\title{
La intercomprensión como herramienta para la integración educativa en el espacio sudamericano: posibilidades, dinámicas y límites a partir de las experiencias del programa PEIF y de la UNILA
}

\author{
Francisco Javier Calvo del $01 \mathrm{mo}{ }^{1}$ (D) @ \\ Angela María Erazo Muñoz ${ }^{2}$ (1) @ \\ ${ }^{1}$ Universidade Federal do Paraná (UFPR), Brasil; ${ }^{2}$ Universidade Federal da Paraíba (UFPB), Brasil
}

Resumen. La primera década del siglo XXI representó para América del Sur una época de expansión económica, de aplicación de políticas de inclusión social y de integración regional. En ese contexto, se implementaron proyectos conjuntos entre los países del Mercosur que promovieron la enseñanza del portugués y del español con el objetivo de formar una ciudadanía latinoamericana plurilingüe. A partir de estas premisas, pretendemos examinar dos modelos educativos implementados en la frontera entre Brasil y los países hispanohablantes: el primero representado por el Programa de Escuelas Interculturales de Frontera (PEIF), destinadas a la educación primaria, y el segundo, por la Universidad Federal de la Integración Latinoamericana (UNILA), dirigido a la enseñanza superior. Inicialmente, describiremos el desarrollo de cada uno de ellos para, a continuación, evaluar sus potencialidades y sus límites. A la luz de estos datos, propondremos líneas de reflexión para proyectos que, en un futuro, pretendan avanzar hacia modelos semejantes de educación plurilingüe. Finalmente, concluimos que la inserción de la intercomprensión representa una herramienta que valoriza el bagaje lingüístico y cultural de los individuos y supera concepciones monolingües. Palabras clave: enseñanza bilingüe; educación intercultural; multilingüismo; intercomprensión.

\begin{abstract}
A intercompreensão como ferramenta de integração educacional no espaço sul-americano: possibilidades, dinâmicas e limites baseados nas experiências do programa PEIF e UNILA

Resumo. A primeira década do século XXI representou para a América do Sul um momento de expansão econômica, a implementação de políticas de inclusão sociale de integração regional. Nesse contexto, foram desenvolvidos projetos conjuntos entre os países do Mercosul que promovessem o ensino de português e de espanhol, com o objetivo de formar uma cidadania latino-americana plurilíngue. A partir dessas premissas, pretendemos examinar dois modelos educacionais implementados na fronteira entre o Brasil e os países hispanofalantes: o primeiro representado pelo Programa de Escolas Interculturais de Fronteira (PEIF), no ensino básico, e o segundo, pela Universidade Federal da Integração Latino-americana (UNILA), voltada ao ensino superior. Primeiramente, descrevemos o desenvolvimento de cada um deles e, em seguida, avaliamos suas potencialidades e seus limites. À luz desses dados, propomos linhas de reflexão para projetos que, no futuro, busquem avançar para modelos semelhantes de educação multilingue. Finalmente, concluímos que a inclusão de intercompreensão é uma ferramenta que promove o acervo linguístico e cultural dos indivíduos e ajuda a superar concepções monolíngües.
\end{abstract}

Palavras-chave: ensino bilíngue; educação intercultural; multilinguismo; intercompreensão.

Intercomprehension as a tool for educational integration in the South American space: possibilities, dynamics and limits based on the experiences of the PEIF program and UNILA

Abstract. The first decade of the 21st century represented for South America a time of economic expansion, of application of policies of social inclusion and regional integration. In this context, joint projects were implemented among the Mercosur countries that promoted the teaching of Portuguese and Spanish with the aim of forming a Latin American multilingual citizenship. Based on these premises, this paper examines two educational models fostered on the border between Brazil and the Spanish-speaking countries: the first represented by the Border Intercultural Schools Program (PEIF), designed for primary education, and the second, aimed at higher education, for the Federal University of Latin American Integration (UNILA). Initially, we will describe the development of each program, in order to evaluate its potentialities and limits. In light of these data, we will propose some guidelines for projects that, in the future, advance towards similar models of multilingual education. Definitely, we conclude that the insertion of intercomprehension (mutual intelligibility) represents a tool that values the linguistic and cultural background of individuals and can efficiently overcomes monolingual conceptions.

Keywords: bilingual education; intercultural education; multilingualism; intercomprehension. 


\section{Introducción: convivencia y contactos de las lenguas románicas en América}

En la actualidad, las Américas concentran el mayor número de hablantes de lenguas neolatinas: aproximadamente 600 millones de personas. Estas poblaciones se localizan de un extremo al otro del continente; desde la Península del Labrador hasta Tierra del Fuego y desde California y los Andes hasta la islas del Caribe. En ese vastísimo espacio geográfico y humano, podemos distinguir varios círculos concéntricos que abarcan países y regiones más o menos amplias como América Latina (considerando los países y comunidades de lengua española, portuguesa y francesa), Iberoamérica (integrada por los países hispanohablantes y por Brasil) e Hispanoamérica (referida solamente a los 19 países hispanohablantes). Además, los movimientos migratorios y las diásporas hacen que las fronteras con la América anglófona, Estados Unidos y Canadá principalmente, sean cada vez más difusas.

En este panorama global, las variedades vernáculas americanas actuales del español, del portugués y también del francés son el resultado de un proceso secular de formación marcado por los contactos lingüísticos de esos idiomas hegemónicos con las lenguas de los pueblos ancestrales, con las lenguas criollas practicadas por las poblaciones de matriz africana y con las que trajeron los emigrantes europeos y asiáticos durante los siglos XIX y XX:

Cet espace de filiation latine qui se dessine aux Amériques est creusé par maintes différences ethniques, géographiques, économiques, religieuses, sociales, politiques, idéologiques, etc. En outre, chez chaque communauté : hispanophone, lusophone et francophone, les langues présentent importantes variations diatopiques, diaphasiques et diastratiques. Une mention spéciale réclame la cohabitation de ces trois langues néolatines avec les langues indigènes, avec les idiomes allochtones apportés par les émigrants européens et asiatiques, avec les créoles; et encore avec des jargons et des codes hybrides comme le spanglish, le portunhol, le lunfardo ou le jopará. (Calvo del Olmo, 2012, p. 402)

Si dirigimos nuestra atención hacia el continente sudamericano, encontramos un elemento que destaca en la configuración lingüística del territorio: la frontera terrestre entre Brasil y sus países vecinos que se extiende a lo largo de más de 15 mil kilómetros ${ }^{1}$. Con la excepción de Surinam, cuya

${ }^{1}$ Ordenados de sur a norte: Uruguai: $1.003 \mathrm{~km}$ de frontera con el Estado Rio Grande do Sul; Argentina: 1.263 km de frontera con los Estados de Paraná (293 km), Santa Catarina $(246 \mathrm{~km})$ y Rio Grande do Sul $(724 \mathrm{~km})$; Paraguay, $1.339 \mathrm{~km}$ de frontera con los Estados de Mato Grosso do Sul (1.131 km) e Paraná (208 km); Bolivia: 3.126 $\mathrm{km}$ de frontera con los Estados de Acre $(618 \mathrm{~km})$, Rondônia $(1.342 \mathrm{~km})$, Mato Grosso (780 km) y Mato Grosso do Sul (386 km); Perú, 2.995 km con los Estados de Amazonas $(1.565$ km) y Acre (1.430 km); Colômbia: 644 km con el Estado de Amazonas; Venezuela, 
lengua oficial es el neerlandés, y de la Guyana (inglés), todos los demás países fronterizos son hispanohablantes. Además, el estado brasileño de Amapá limita con la Guayana Francesa, región dependiente políticamente de Francia, 655 km que representan la frontera más extensa de la República Francesa. Esta contigüidad geográfica permite contactos entre las comunidades a ambos lados de los límites políticos; sin embargo, las agendas de dichos países no siempre han priorizado la cooperación mutua y, en diversas épocas, han dirigido su atención hacia los países del norte global, principalmente a los Estados Unidos y a Europa. Por todo ello, consideramos que aun hoy sigue siendo necesario pasar de la mera contigüidad lingüística y cultural a la continuidad (Escudé y Janin, 2010, p. 77) que permita el tránsito de los ciudadanos entre esas lenguas pertenecientes al diasistema románico. De hecho, el potencial científico, cultural, político y económico de los contactos entre Brasil y los países hispanohablantes viene siendo tema de estudio en las áreas de las humanidades y de las ciencias sociales.

La reflexión que esperamos desarrollar en los próximos puntos pretende pasar de la contigüidad, descrita por Moreno Fernández, a la continuidad en Latinoamérica, lo cual permitiría superar la hegemonía de un modelo monolingüe y articular nuevas formas de pluralismo lingüísticocultural. Como apunta García (2011, p.31), las lenguas han asumido un rol central en un orden mundial que avanza hacia la multipolaridad. En este sentido, los modelos educativos pueden desempeñar un papel importante en favor de la difusión del plurilingüismo y de la promoción del intercambio entre lenguas, saberes y culturas como sugieren Barrios (2006) y Hamel (2013). Es decir, la educación es una palanca promotora de cambios y una herramienta muy valiosa para la formación de la ciudadanía. Partiendo de todos estos presupuestos, dedicaremos las próximas secciones de este trabajo a presentar y examinar sucintamente dos experiencias que se han desarrollado durante las dos primeras décadas del presente siglo: el Programa de Escolas Interculturais de Fronteira (PEIF), enmarcado en las políticas lingüísticas del Mercosur, y la Universidade Federal para a Integração Latino-Americana (UNILA), que igualmente propone un modelo de integración académica y lingüística en ámbito latinoamericano. Trataremos de describir algunas líneas comunes entre ambas experiencias más allá de las especificidades y diferencias que necesariamente presentan y propondremos la intercomprensión como un abordaje que puede resultar eficaz para superar los impasses en los que actualmente se encuentran.

$1.492 \mathrm{~km}$ totales repartidos entre los Estados de Roraima (954 km) y Amazonas (538 km); la Guyana: 1.606 km con los Estados de Pará (642 km) y de Roraima (964 km); Surinam: 593 km de frontera con los Estados de Amapá (52 km) y de Pará (541 km) y la Guayana Francesa: 655 km con el Estado de Amapá. 


\section{El Programa de Escuelas Interculturales de Frontera (PEIF) como motor de la integración regional en la educación primaria: orígenes, desarrollo y obstáculos.}

Desde la década de 1990, los planes de acción del Sector Educativo del Mercosur (SEM)² vienen recogiendo la difusión de la enseñanza del español y del portugués como una cuestión prioritaria que se traduce tanto en la introducción de ambas lenguas en los diferentes sistemas educativos y niveles como en la formación de profesores de portugués y de español, preferentemente de manera integrada. El objetivo que las autoridades esperan con tales acciones es fortalecer los vínculos regionales, fomentar los intercambios académicos y mejorar la calidad de la educación en la región. En este contexto, surgió el Programa PEIF/PEIBF cuyos puntos principales examinaremos a lo largo de esta sección.

Como explican Sagaz (2013) y Lorenzetti y Torquato (2016), originalmente el programa recibió el nombre de Escolas Interculturais Bilíngues de Fronteira (PEIBF) y, en un primer momento, tomó la forma de un acuerdo bilateral firmado entre el Ministerio de Educación, Ciencia y Tecnología (Argentina) y el Ministério da Educação (Brasil) con el propósito de construir una ciudadanía regional bilingüe e intercultural, fomentando así una cultura de paz y de cooperación transfronteriza. La declaración conjunta fue firmada en 2003 en Brasilia y ratificada en la primera reunión técnica bilateral que tuvo lugar en diciembre de 2004, en Buenos Aires. Esto representó un hito significativo en la cooperación entre los dos vecinos del cono sur:

A Declaração Conjunta de 2003, ratificada em 2004, na cidade de Buenos Aires, pelos então Ministros da Educação da Argentina, Daniel Filmus e do Brasil, Tarso Genro, indica a criação, em região de fronteira, de três escolas em cada país com modelo bilíngue intercultural e dispõe a seguinte ação para imediata implementação. (Sagaz, 2013, p. 38)

De esta forma, el programa inició oficialmente con dos escuelas públicas: una en la localidad de Paso de los Libres, provincia de Corrientes, fronteriza con la ciudad brasileña de Uruguaiana, estado de Rio Grande do Sul, y otra en Bernardo de Yrigoyen, Misiones, fronteriza con los municipios brasileños de Dionísio Cerqueira, Santa Catarina, y Barracão, Paraná. Al año siguiente, en 2006, las localidades de Puerto Iguazú (Misiones) y Foz do Iguaçu (Paraná), Santo Tomé (Corrientes) y São Borja (Corrientes), y La Cruz (Corrientes) e Itaqui (Rio Grande do Sul) se sumaron al proyecto. Cabe decir que estas localidades conformaban áreas urbanas binacionales siendo

${ }^{2}$ Las informaciones detalladas de los planes de acción por cuatrienios pueden ser consultadas en la página web de la institución http://edu.mercosur.int/es-ES/. 
separadas por un puente, una avenida o unos pocos kilómetros por lo que el contacto entre las poblaciones argentinas y brasileñas ya existía previamente y las comunicaciones eran relativamente sencillas. Las escuelas de los dos lados de la frontera deberían funcionar como una unidad operativa que recibía el nombre de escuelas-espejo/escolas-espelho. Con ello se pretendía que las dos instituciones actuaran conjuntamente y sumaran sus esfuerzos en la construcción de la modalidad de educación intercultural y bilingüe. Lo cual, no obstante, enmascaraba las desigualdades socioeconómicas que muchas veces existían, así como otros factores de planificación como los períodos de vacaciones y días festivos o, incluso, la diferencia de huso horario durante el periodo estival:

O Documento Básico menciona que "há uma caminhada de médio prazo até que se possa pensar tanto em um modelo comum compartilhado quanto em um calendário único [...]" (BRASIL, 2008, não paginado). Salientamos que já havia três anos que o PEIBF estava em implantação sem contemplar um calendário favorável às práticas conjuntas, a saber: planejamento conjunto; cruce com vistas ao um modelo de educação bilíngue; metodologia de ensino por projetos de aprendizagem conjuntos. (Sagaz, 2013, p. 131)

A pesar de esas deficiencias de planificación, en 2007, equipos técnicos binacionales elaboraron un documento marco: Modelo de ensino comum de zona de fronteira, a partir do desenvolvimento de um Programa para a educação intercultural, com ênfase no ensino do português e do espanhol con el fin de reforzar y articular el Programa que entonces pasó a Ilamarse Escolas Interculturais de Fronteira (PEIF) ${ }^{3}$. Más tarde, la base legal del programa PEIF en Brasil fue completada a través Documento Marco Referencial de Desenvolvimento Curricular, redactado y aprobado en el seno del Mercosur, y por la Portaria dn. 798 del Ministerio de Educación brasileño del 19 de junio de 2012. Con todo ello se pretendía organizar y fomentar "a interação entre os agentes educacionais e as comunidades educativas envolvidas, de tal maneira a propiciar o conhecimento do outro e a superação dos entraves ao contato e ao aprendizado" (MEC y MECyT, 2008, p. 21).

En aquel momento, también se hizo latente la necesidad de documentar la realidad sociolingüística de los profesores y los estudiantes que participaban en el programa focalizando la investigación en tres cuestiones: “a) el grado de conocimiento del español estándar de los docentes de las

${ }^{3}$ La información a la que hemos tenido acceso en las páginas oficiales no permite describir de forma conclusiva los motivos epistemológicos o metodológicos que sustentaron el cambio en la denominación oficial del programa pasando de PEIBF para PEIF. Sin embargo, es posible que el reconocimiento del carácter multilingüe social y geográfico del entorno haya llevado a los gestores a reformular el nombre de forma más internacional e inclusiva. 
escuelas de frontera de Corrientes y Misiones; b) el grado de conocimiento de portugués de docentes y alumnos y c) las representaciones sobre estas lenguas y otras, como el guaraní" (MERCOSUR, 2007, p. 6). Las informaciones de la página del MEC permiten imaginar la dinámica de los grupos que participaban en el PEIBF:

Às terças e quintas-feiras, cerca de 80 estudantes do primeiro ao quinto ano do ensino fundamental da escola estadual Dr. Theodureto Carlos de Faria Souto, situada em Dionísio Cerqueira (SC), têm aulas em espanhol com professores argentinos. Ao mesmo tempo, 150 alunos da Escuela 604 em Bernardo de Irigoyen, na província de Misiones, Argentina, são ensinados em português por docentes brasileiros. Mas a troca de experiências vai muito além da língua. Inseridas no Programa Escolas Interculturais de Fronteira (Peif), as escolas têm enfoque no conhecimento cultural mútuo. (Tancredi, 2015)

Así pues el objetivo era mejorar la integración regional de las nuevas generaciones por medio de la educación intercultural, como se afirma en el portal del MEC: "Seu principal objetivo é o de promover a integração regional por meio da educação intercultural que garanta formação integral às crianças e aos jovens nas regiões de fronteira do Brasil com outros países" (Brasil, s.f.). A este primero se sumaban otros objetivos específicos:

a) uso da segunda língua de maneira que esta passe a estar cada vez mais presente no cotidiano da escola, de forma oral e escrita, por meio de uma relação com um falante nativo da língua objeto do ensino-aprendizagem;

b) relação pessoal/profissional com falantes nativos cujo contato permite conhecer e vivenciar o sistema escolar do país vizinho;

c) ampliação da base informacional dos conteúdos escolares, deixando de focar unicamente o nível nacional e ocupando-se também com a Região como unidade de trabalho (Brasil, s.f.).

El español y el portugués funcionaban como vectores para la consecución de tales objetivos y como herramientas para la construcción de saberes disciplinarios. No se trataba sólo de enseñar español y portugués sino de enseñar en español y en portugués considerando el bagaje lingüístico y cultural del profesor y de los alumnos. En consecuencia, era necesario contemplar las variedades vernáculas practicadas por las poblaciones de frontera y la presencia del guaraní, que también es oficial en el Mercosur. Cabría también apuntar las variedades de italiano, de alemán y de polaco practicadas como lenguas heredadas en ese ámbito geográfico como otros componentes lingüísticos y culturales pasibles de valorización. 
En todo caso, el PEIF proporcionó un entorno real de bilingüismo para el conjunto de la comunidad académica; así lo recoge la página que hemos citado anteriormente a partir del testimonio de unos de los profesores que participaron:

A língua é um detalhe, mas a base é a interculturalidade; trabalhamos os costumes dos dois países, a fauna, a flora, o turismo, a alimentação", explica Mauro Prado, diretor da escola catarinense. 0 principal benefício, segundo ele, é a aproximação entre os dois países, por meio da educação. "A fronteira é tão perto, mas o conhecimento da realidade de cada um acaba sendo tão distante, e é aí que entram as vantagens do programa (Tancredi, 2015)

Dentro del desarrollo del PEIF, las universidades tenían un papel importante pues les correspondía la formación de los profesores. Las actividades didácticas se planificaban de manera conjunta entre los docentes de las escuelas-espejo y el apoyo pedagógico de equipos universitarios. En un primer momento, la asesoría pedagógica y la formación continuada para profesores brasileños, argentinos y paraguayos estuvo a cargo del Instituto de Investigação e Desenvolvimento em Política Lingüística (IPOL), con sede en la ciudad de Florianópolis, capital del Estado de Santa Catarina pero, a partir de 2012, dicha asesoría fue transferida a varias universidades próximas a las ciudades donde se encontraban las escuelas participantes en el PEIF: como la Universidad Federal de la Frontera Sur (UFFS) o la Universidad para la Integración Latino-Americana (UNILA), de la que hablaremos en detalle en los próximos apartados. También el Instituto Misiones de Argentina colaboró en la formación. Los cursos de Formación del Programa Escuelas Interculturales de Frontera, Ilamado PROPEIF, recibió el incentivo económico de las universidades cuyos docentes, con el apoyo de alumnos becarios, deberían preparar a los profesores de las escuelas para elaborar proyectos de aprendizaje que contemplaran las relaciones históricas, sociales, económicas y culturales de las áreas de frontera. La propuesta del PROPEIF también preveía organizar un repositorio con esas formaciones Ilamado Centro de Memorias, que daría continuidad y visibilidad a dichas acciones.

A diferencia de otras propuestas de educación intercultural en América Latina como la etnoeducación (Colombia) o las escuelas indígenas interculturales (México, Bolivia, Perú), que solo se centran en el desarrollo de propuestas interculturales dentro del país, el PEIF permitió traspasar los límites geográficos pensando en fortalecer lazos para una ciudadanía transnacional y el desarrollo de una conciencia plurilingüe.

En los años sucesivos se incorporaron otros municipios de los países limítrofes llegando a abarcar (además de a la Argentina) Paraguay, Uruguay, Bolivia y Venezuela. Además de los que hemos mencionado anteriormente, 
Sagaz (2013, p. 113) presenta datos procedentes del IPOL sobre tres nuevas escuelas que se habían incorporado en 2008: 1) Ponta Porã en Mato Grosso con la escuela-espejo de la localidad paraguaya de Pedro Juan Caballero; 2) Jaguarão, Rio Grande do Sul, y Río Blanco, Uruguay; Chuí, Rio Grande do Sul, con la homónima ciudad uruguaya de Chuy; 4) Pacaraima, Roraima, y Santa Helena de Uairén, Venezuela. La noticia publicada el 22 de mayo de 2014 apuntaba en esa misma dirección.

O Programa Escolas Interculturais de Fronteira passa por um processo de expansão, podendo alcançar 327 escolas já pré-selecionadas, localizadas em 36 municípios em área de fronteira, nos estados do Acre, Amazonas, Amapá, Mato Grosso do Sul, Paraná, Rondônia, Roraima, Rio Grande do Sul e Santa Catarina. De acordo com o Censo Escolar 2012, as 17 escolas do PEIF atendiam à época 7.522 estudantes no ensino fundamental. 0 programa integra a política nacional de educação integral e se desenvolve no âmbito do programa Mais Educação (Brasil, 2014).

Si bien todos los documentos examinados coinciden en reconocer la importancia del programa PEIF como motor de integración regional, también es evidente que hubo carencias que lastraron su desarrollo desde el origen; principalmente debidas a la falta de partidas presupuestarias propias y la adecuada remuneración de las y los docentes, para quienes este trabajo no debería convertirse en una carga suplementaria. Como apuntan Lorenzetti y Torquato (2016), la ampliación del programa a nuevos estados y países se hizo sin haber resuelto tales cuestiones fundamentales. Esto constituía un punto flaco para darle continuidad y, de hecho, la tendencia de expansión comenzó a frenarse en 2015, justamente al cumplirse diez años desde el inicio del programa. El clima de inestabilidad política generado en la región, a raíz del proceso de impeachment de la presidenta Dilma Rousseff en 2016 así como el cambio de prioridades en las agendas nacionales y la discontinuidad de los actores políticos, repercutió negativamente en las acciones del PEIF, cuyos resultados se proyectaban a largo plazo. Cabe decir que las informaciones disponibles en las páginas web de los ministerios y de otros órganos públicos son fragmentarias y algunas de ellas no están más en línea, por lo que el registro y la explicación cabal de este proyecto se hace complicada para la investigación. En las entrevistas realizadas por Lorenzetti y Torquato (2016, p. 99) "os docentes assinalam o risco de toda a experiência intercultural e pedagógica ser esquecida uma vez que as ações do Programa, em cada um dos países, caminham isoladamente, independentemente do país 'parceiro'". En ese sentido, podemos afirmar que se ha producido un abandono del PEIF por parte de las autoridades políticas y educativas que tenían la responsabilidad sobre la implementación y manutención del mismo. Pese a ello, el Programa PEIF continúa despertando el interés académico como precedente que podría ser reconsiderado, con sus puntos fuertes y débiles, 
a la hora de realizar acciones futuras. Así pues, nos parece que la tarea de documentación y difusión son fundamentales y esperamos, en la medida de nuestras posibilidades, haber da una pequeña contribución con estas páginas.

\section{La Universidad Federal para la Integración Latinoamericana (UNILA): posibilidades y límites de un proyecto de universidad bilingüe y multicultural en América Latina}

Una vez superado el Ilamado Consenso de Washington que imperó en las relaciones internacionales latinoamericanas durante la década de 1990, se asiste en los primeros años del siglo XXI al fortalecimiento de bloques regionales de cooperación y movilidad como el Mercosur, la Unasur y la CEPAL. Como ya hemos mencionado, la educación ocupa un espacio relevante en las agendas de los procesos de integración regional, principalmente a través del Mercosur Educativo. Es así como en 2008, se establece el programa Universitario Mercosur con la intención de apoyar la movilidad de estudiantes y docentes en la educación superior. Esta demanda surge de "la necesidad de una estructura que permita conectar los resultados de la enseñanza y de la investigación con las necesidades reales de la integración y del desarrollo de las sociedades suramericanas" (Briceño Ruiz, 2011, p. 251).

Al avanzar en los objetivos de integración y de movilidad, surgió una primera propuesta para la fundación de una universidad que impulsada por la mencionada entidad supranacional. Sin embargo, varios contratiempos de índole económica, política y administrativa impidieron que el proyecto de la creación de la universidad no fuera aprobado por el conjunto de los países miembros del Mercosur. En ese momento, el Ministerio de Educación (MEC) de Brasil tomó la iniciativa de presentar el proyecto de ley para la creación de la Universidade Federal da Integração Latino-Americana (UNILA); el cual fue probado por el Congreso en Brasilia en 2009 y sancionado por el entonces Presidente de la República de Brasil, Luiz Inácio Lula da Silva. La bonanza económica que vivía Brasil durante dicho período y el compromiso del gobierno de Lula con la educación superior (a través de los programas ProUni e ReUni), fueron factores determinantes en la ejecución del proceso de implantación de la nueva universidad. La UNILA fue inaugurada en 2010 en el municipio de Foz do Iguaçu, Estado de Paraná, situado en la Ilamada triple frontera Brasil, Argentina (Puerto Iguazú, provincia de Misiones) y Paraguay (Ciudad del Este). A la luz de los documentos oficiales que describen este proyecto, la UNILA se edifica sobre los principios filosóficos y metodológicos formulados para direccionar la gestión universitaria y sus 
prácticas en la enseñanza, la investigación y la extensión. Estos principios son: interdisciplinariedad, interculturalidad, bilingüismo, integración solidaria y gestión democrática (UNILA, 2013, p. 15).

Observamos que entre estos componentes se menciona explícitamente el carácter bilingüe en portugués y español de la institución. Es decir, los estudiantes y profesores brasileños e hispanoamericanos que acoge la institución tienen el derecho de usar para sus actividades académicas y profesionales la lengua que prefieran. Además, todos los estudiantes tienen la obligación de cursar un ciclo común durante los tres primeros semestres que, entre otros componentes académicos, ofrece clases de español para los brasileños y de portugués para los hispanohablantes (entre ocho y seis horas semanales dependiendo de los planes de estudio). Ello, a priori, ofrece un terreno amplio para la observación de prácticas comunicativas plurilingües y de la intercomprensión en lenguas románicas en ámbito académico como ha sido apuntado por Degache y Garbarino (2017) ${ }^{4}$. Sin embargo, el estudio de Erazo Muñoz (2016) mostró ciertas divergencias entre la documentación institucional y las prácticas comunicativas cotidianas por el contexto geográfico (la universidad se encuentra en territorio brasileño), el rol de los hablantes en la institución y su identificación con código lingüístico. Además estos actores provienen de diversos ambientes socioculturales que determinan el proceso de aprendizaje en lenguas y su inserción en la cultura académica.

El mismo trabajo concluye que, a pesar de las dificultades, en las interacciones entre hablantes de diferentes lenguas, la intercomprensión es un instrumento de comunicación y de transmisión de saberes privilegiado en este entorno. También destacó una serie de aspectos relevantes: a) en espacios formales, el español y el portugués eran las lenguas principales de uso, en concordancia con las directivas institucionales; b) en un ambiente bilingüe de aprendizaje se daba una diversidad de combinaciones posibles para el uso de las lenguas de enseñanza, a la que se sumaban también otras formas de lenguaje, alternancia de lenguas y situaciones de interlengua como el portuñol; c) este contexto permite una gran flexibilidad en la comunicación; d) se evidenció una cierta oposición entre los componentes curriculares universitarios lingüísticos y no lingüísticos.

${ }^{4}$ Existen diversas instituciones bilingües en el espacio de la enseñanza superior. Las universidades de Catalunya, Valencia y Baleares son bilingües (catalán y español), así como las de Galicia (gallego y español) y del País Vasco (euskera y español). En Quebec y en Canadá algunos establecimientos implementan estancias bilingües (francés e inglés). Experiencias de este tipo también existen en las universidades de algunos cantones suizos (francés y alemán). De la misma forma, las universidades de los países del Magreb, Marruecos, Argelia y Túnez, funcionan en francés y en árabe. 
La inscripción de la UNILA en el contexto nacional (Brasil) y local (Foz do Iguaçu) afecta directamente el equilibrio del uso y de la percepción de las dos lenguas (español y portugués) y de sus hablantes, ya que la situación de los hispanohablantes se aproxima más a la situación de aprendizaje de una L2 en inmersión, mientras que los brasileños se encuentran en situación de aprendizaje de una lengua extranjera, a pesar de la proximidad con usuarios y territorios de lengua española. En otras palabras, los datos etnolingüísticos indican que las relaciones entre lenguas y hablantes son heterogéneas y constatan situaciones asimétricas que se encajan en la descripción de una situación diglósica. Este término implica una situación conflictiva para autores como Lüdi y Py (2003), y permite explicar la distribución jerárquica de las funciones de dos o más lenguas en una comunidad. Las mencionadas relaciones asimétricas están presentes en el contexto de la UNILA y se reflejan principalmente en el uso preferente e, inclusive, en la imposición del portugués como lengua de comunicación privilegiada en el contexto académico e institucional. En suma, al no ofrecer una política y un planeamiento lingüístico explícito y coherente, la responsabilidad del mantenimiento del bilingüismo institucional de la UNILA, la planificación y el uso lingüístico se ven delegados en los hablantes, de manera particular, en los profesores y estudiantes.

Más allá de la dicotomía portugués/español, la comunidad de docentes y estudiantes que integra la UNILA posee competencias lingüísticas y culturales enormemente diversas. Primeramente, el análisis del componente étnico y de las nacionalidades de la población pone de manifiesto la presencia de un número importante de lenguas indígenas como el guaraní, el quechua y el aimará. Por otro lado, profesores y alumnos que han realizado parte de su formación en el extranjero conocen lenguas occidentales como el inglés, el italiano, el francés y el alemán. Visto desde la perspectiva de la diglosia, todos estos repertorios no encuentran espacios de valorización, puesto que no existen directrices institucionales que permitieran invertir la tendencia y potenciaran estos conocimientos en favor de la construcción de una universidad plurilingüe.

En síntesis, el estudio de Erazo Muñoz (2016) constata diversas manifestaciones de práctica plurilingüe en el contexto de la UNILA; entre las cuales, destaca el papel de la intercomprensión como una forma de comunicación y medio de instrucción recurrente. No obstante, ese mismo trabajo pone de manifiesto las asimetrías entre el poder de las lenguas ya que el portugués está amparado por las leyes nacionales brasileñas (la UNILA no deja de ser una de las 68 universidades federales) y justificado por la alta presencia de profesores, alumnos y cuerpo técnico brasileños frente a una población hispanohablante minoritaria constituida solo por alumnos y profesores, todos conocedores de la lengua portuguesa y de la cultura académica 
brasileña por estar en un ambiente de inmersión. Además, dichas asimetrías no solo se reflejan en el uso de las lenguas sino que también se traducen en las relaciones de estatus social y de poder de los usuarios.

A nivel macro se constata, desde 2015, una reorientación geopolítica de América del Sur como resultado de la hegemonía conservadora en Argentina y en Brasil, que subordinó la política exterior de los países a los intereses estadounidenses. A ello se suma un panorama de crisis económica que es utilizada para justificar cortes na educación.

Por todo ello, en los últimos años, las becas para los estudiantes han disminuido como consecuencia de cortes presupuestarios, lo que se traduce en una reducción del número de estudiantes hispanos, para quienes los desplazamientos y el coste de la vida en Foz do Iguaçu resultan ser mucho más elevados. Por otro lado, en 2015 se realizó una selección de ingreso destinada a jóvenes haitianos residentes en Brasil ${ }^{5}$, y en 2019 se abrieron dos convocatorias destinadas a estudiantes indígenas de varios países de la región y otra para refugiados. Si bien los repertorios lingüísticos como hablantes de creole ayisyen y francófonos de esta comunidad amplían las lenguas de la universidad, la integración académica es mediada por el portugués.

A pesar de las dificultades las fases iniciales de una institución en formación (como la falta de un campus propio y de efectivos en el cuerpo docente) y de ciertos ataques sufridos en 2017 (cuando un diputado del PMDB de Paraná presentó la Medida Provisória 785/17, que pretendía transformarla en la Universidade Federal do Oeste do Paraná, desfigurando el proyecto de integración regional que le dio origen) la UNILA completará el año que viene su primera década de existencia. Los números actualizados de estudiantes (alrededor de 6.000 entre grados y posgrados) y docentes (362) muestran cifras de una universidad modesta pero consolidada ${ }^{6}$. Consideramos que la situación de frontera, la llegada a cada año de un grupo representativo de alumnos hispanohablantes y el paraguas del bilingüismo oficial garantizan la presencia de la lengua española en ese ambiente. No obstante, el portugués continúa teniendo un peso central y el español es usado en espacios reducidos. De este modo, continúa siendo necesario diseñar una política lingüística que planifique el papel de cada lengua, que vele por el cumplimiento de las directrices y que compense los desequilibrios. Esta además debería abarcar los demás repertorios lingüísticos presentes entre los miembros de la comunidad académica.

${ }^{5}$ Véase la información sobre el Programa Pró-Haiti en la página web de la institución: https://unila.edu.br/noticias/pro-haiti-5

${ }^{6}$ Las informaciones completas están disponibles en la página web de la institución. Recuperado de https://portal.unila.edu.br/secom/imprensa/numeros. 


\section{La inserción de la intercomprensión en las universidades latinoamericanas: ¿una vía para la promoción de plurilingüismo en ámbito académico?}

A partir de los dos programas que presentamos en las secciones anteriores, pretendemos reflexionar sobre las prácticas plurilingües e intercomprensivas en ambientes académicos. En este sentido, debemos recordar que la UNILA y el Programa PEIF se encuadran dentro del espacio político del Mercosur y responden, lato sensu, a la voluntad de acercar a los ciudadanos latinoamericanos y promover los intercambios educativos y el conocimiento lingüístico y cultural entre los países vecinos. Sin embargo, hay diferencias importantes entre ellos puesto que la creación de la UNILA fue el resultado de una decisión unilateral del Ministerio de Educación Brasileño, mientras que el programa PEIBF resultó de los acuerdos bilaterales entre Argentina y Brasil, dirigidos más tarde al conjunto del Mercosur mediante el PEIF. Los avatares políticos sucesivos condicionaron el desarrollo de todos ellos a largo plazo. En este punto, un elemento especialmente negativo fue la promulgación de la ley n. ${ }^{\circ} 13.415$ en 2017 por parte del poder legislativo brasileño, que revocó la Ley . $^{\circ} 11.161$ de 2005, esta, a su vez, había instituido la oferta obligatoria de lengua española en el llamado "ensino fundamental" (que abarca de los seis a los quince años de edad) a nivel federal. Como explica Ferreira (2018, p. 54) el documento de la Base Nacional Comum Curricular sustituyó el nombre de la asignatura "língua estrangeira moderna" por el de "língua inglesa" lo que en la práctica se traduce por la hegemonía del inglés en el currículum escolar de Brasil y la exclusión de las demás lenguas.

Sin pretender ser una solución a todas estas cuestiones que dependen, en gran medida, de instancias políticas nacionales y de las correspondientes partidas presupuestarias, la intercomprensión entre lenguas próximas -tal y como ha sido descrita en el Marco de Referencia para los Enfoques Plurales de las Lenguas y de las Culturas, MAREP (Candelier et al., 2012)-, y más específicamente la intercomprensión en lenguas románicas (ICLR), puede ser una herramienta para salir de este impase promoviendo la formación en lengua materna mediante el contacto y la integración con otras lenguas próximas ${ }^{7}$. Cabe decir que circulan varias definiciones del término intercomprensión, formuladas desde supuestos teóricos y metodológicos diferentes y complementarios.

${ }^{7}$ Existen algunas investigaciones experimentales realizadas en ese contexto como la de Dautzenberg (2016) con alumnos de once y doce años en una escuela de la periferia de la ciudad de Curitiba y la de Felix (2016) con estudiantes de una aldea indígena mbya, clasificada dentro del grupo étnico guaraní, del Estado de Paraná. 
Dentro del contexto académico latinoamericano, la intercomprensión ofrece una serie de posibilidades que, a nuestro juicio, son valiosas. La intercomprensión responde a esas necesidades de los individuos, abriéndoles la posibilidad de circular entre lenguas, variedades y registros para atender a sus propósitos comunicativos, negociar significados y compensar las asimetrías entre lenguas y hablantes. En este punto, hay que recordar que la proximidad entre dos lenguas no es suficiente para que automáticamente se produzca la comunicación en intercomprensión, sino que es necesario que exista la voluntad y la disposición de los hablantes, es decir, la intencionalidad de los mismos (Escudé y Janin, 2010, p. 40), así como un conjunto complejo de procedimientos para la movilización de recursos lingüísticos, cognitivos y sociales, que posibiliten que esta comunicación sea percibida y evaluada como eficaz.

Además, la práctica de la intercomprensión despierta en los propios hablantes una conciencia plurilingüe, una experiencia de comunicación integradora de las diferencias y las particularidades de esas culturas en contacto. En otras palabras, la intercomprensión supera la dicotomía bilingüe de las normas estandarizadas del español y del portugués, y abre un espacio para otras lenguas y variedades que han estado presentes en el territorio latinoamericano:

Asimismo la ICLR [intercomprensión en lenguas románicas] representa una práctica constitutiva del espacio latinoamericano. El proceso colonizador, iniciado en 1492, provocó que tres lenguas neolatinas - el español, el portugués y el francés - se convirtieran en idiomas hegemónicos de la región; donde, hasta el día de hoy, conviven con las lenguas ancestrales de los pueblos indígenas, las lenguas criollas (nacidas del propio contacto entre poblaciones africanas esclavizadas y colonizadores europeos) y las lenguas alóctonas (traídas por los inmigrantes en el siglo XIX y XX). De todo ello, resultan diversos contextos de bilingüismo, diglosia y contactos que, en ocasiones, pueden dar lugar a la aparición de códigos híbridos como el spanglish (español-inglés), el portunhol (portugués-español), el cocoliche (italiano-español), la media lengua de Ecuador (quechua-español) o el jopará (guaraní-español) (Calvo del Olmo, 2018, p.11-12).

Todas estas lenguas pueden incluirse en las dinámicas de intercomprensión, lo cual no solo permitiría la promoción del plurilingüismo sino también un proceso de ciudadanía, respeto y sensibilización hacia aquellas voces que estuvieron largo tiempo silenciadas, negadas y prohibidas en los espacios públicos. En este sentido,recordamos la posición de Hamel (2008):

La nueva relación que emerge entre el español y el portugués como lenguas de integración regional en el Mercosur refleja de hecho una orientación que pretende ampliar y potencializar los "campos enunciativos" (Guimarães, 1999) de ambas lenguas y crear un bi-plurilinguismo aditivo. Cualquier 
política lingüística que impulse este objetivo no podrá ceñirse a las tradicionales políticas homogeneizadoras del Estado Nacional que se limitaron a estandarizar las lenguas en sus territorios nacionales (Hamel, 2008, p. 73).

No es de extrañar, por tanto, que la ICRL haya despertado el interés de profesores e investigadores en universidades latinoamericanas. Citamos aquí las experiencias de la Universidad Nacional de Córdoba (UNC) en Argentina y de la Universidad de Playa Ancha (UPLA) en Chile, cuyos equipos desarrollaron sendos métodos de intercomprensión: InterRom (Carullo et al., 2007) e Interlat (Tassara y Moreno, 2007). A su vez, los equipos de la UDELAR han contribuído a la creación de materiales en plataformas como itinerarios romances y Limbo (Unión Latina). En Brasil, varias universidades han Ilevado a cabo formaciones en intercomprensión; algunas de ellas incluso han implementado asignaturas en sus respectivos currículos como la USP y la UNICAMP en el Estado de São Paulo, y las federales de Minas Gerais (UFMG) en Belo Horizonte, de Paraná (UFPR) en Curitiba, en Campina Grande (UFCG) y Rio Grande do Norte (UFRN) además de la propia UNILA. Asimismo, la Universidad de Antioquia (UDEA) en Colombia propone una formación en ICRL para los alumnos de maestría. El siguiente mapa permite localizar todas las instituciones referidas, ello no significa que no existan otras universidades y órganos educativos que estén pensando actividades dentro de este abordaje en la región.

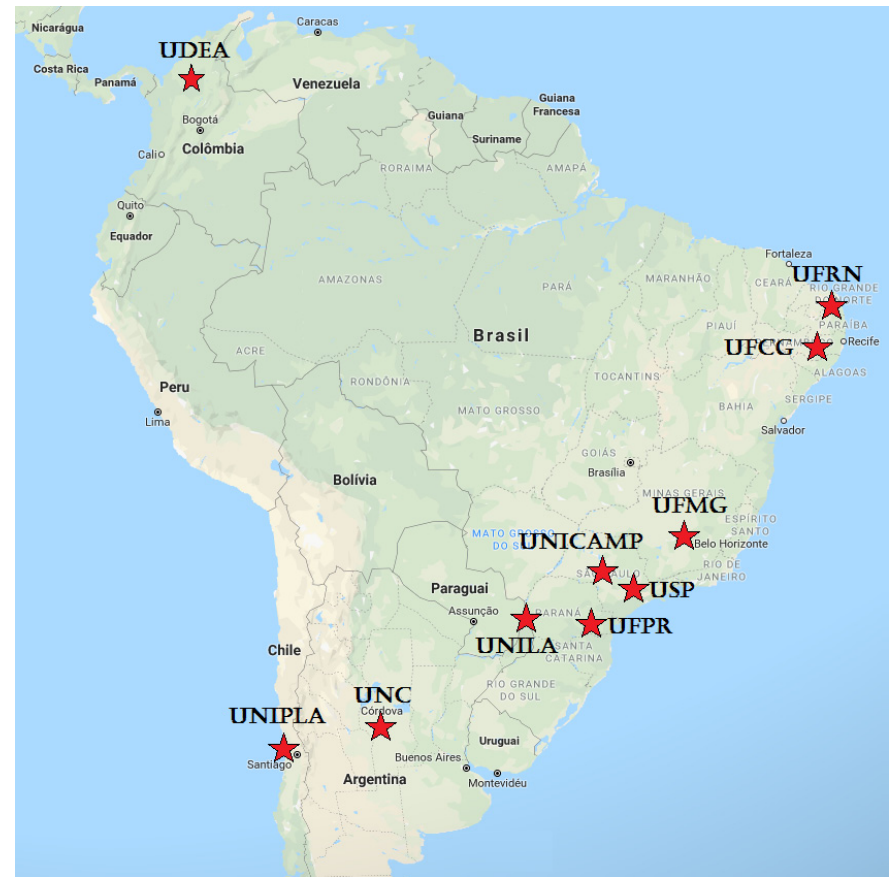

Fuente: Elaboración de los autores. 
Estas temáticas concentran un gran potencial didáctico entendiendo las lenguas como espacios de construcción de saberes. Desde esa perspectiva, la intercomprensión favorece la adquisición de contenidos; así, no se trataría de formar en intercomprensión sino de formar a través de la intercomprensión. Esa posibilidad puede ser denominada como intercomprensión integrada: "Cette approche plurilingue a l'avantage de mettre en rapport le travail à partir de plusieurs langues proches avec des contenus scolaires relevant de différents domaines disciplinaires (mathématiques, sciences, histoire, etc.)" (Gajo y Fonseca, 2014, p. 95). Cabe recordar que este era el espíritu del Programa PEIF y que podría ser retomado en futuros proyectos dirigidos a los distintos niveles de enseñanza.

En síntesis, están aumentando las necesidades de abrir espacios de intercambio económico, social y cultural, que promuevan la movilidad y la cooperación; pero, para que estas propuestas se lleven a cabo, es indispensable crear puentes de comunicación y comprensión mutuas. El diseño e implantación de políticas lingüísticas coherentes tienen una importancia crucial en la consecución de dicho fin. Asimismo, el registro y análisis de las experiencias precedentes proporciona elementos valiosos para cualquier acción que se proponga avanzar en dicha dirección.

\section{Conclusiones}

Autoras como García (2011) y Kramsch (2014) subrayan el papel fundamental del lenguaje y de la enseñanza de las lenguas en el contexto globalizado y multipolar. En este sentido, las experiencias de los programas que presentamos a lo largo de estas páginas muestran el potencial de aqueIlas iniciativas que fomenten el conocimiento mutuo para la construcción de una ciudadanía latinoamericana y el desarrollo del bilingüismo activo portugués-español. PEIBF/PEIF y UNILA han sido, y continúan siendo, una referencia importante a nivel regional y estudiarlos nos permite apuntar hacia nuevas reflexiones que puedan enriquecerlos, adecuarlos a los desafíos políticos y económicos cambiantes y garantizar su continuidad a largo plazo. Sin embargo, también existen lagunas que se traducen en la ausencia de lineamientos lingüísticos y pedagógicos coherentes y en la falta de recursos económicos que permitan una implementación eficaz. En todo caso, y pese a los límites y carencias apuntados, nos parece esencial que estos programas tengan continuidad pues han significado un esfuerzo y una inversión importante para la región. 
En un panorama político marcado por la inestabilidad, es necesario reflexionar en conjunto sobre los rumbos de un espacio de educación integrada. Una de las posibilidades que podríamos apuntar, en el caso del PEIF, así como dentro de la educación primaria en Brasil y en los países limítrofes, es la inclusión de actividades plurilingües a partir de la intercomprensión integrada de lenguas y contenidos (Gajo y Fonseca 2014). De hecho, vienen realizándose experiencias en esta dirección en el Estado de Rio Grande do Norte (Alas-Martins, 2014; Pinheiro-Mariz, 2017; De Sousa, 2019 ) y en la provincia de Córdoba en Argentina (Pérez y Marchiaro, 2016; Pérez, 2017); regiones por tanto no limítrofe. Ello pone de manifiesto la viabilidad de este tipo de acciones con resultados prometedores. En otras palabras, las experiencias de bilingüismo español-portugués y la introducción de elementos multiculturales representan una base sólida para el desarrollo de una discusión teórica sobre la intercomprensión en el contexto latinoamericano.

Por otro lado, aunque los políticas de internacionalización otorguen prioridad a la enseñanza en y del inglés en los niveles de la enseñanza superior, las acciones específicas tienden a flexibilizar cada vez más las prácticas lingüísticas. Consciente de que la calidad de enseñanza y la investigación científica están vinculadas a la política lingüística universitarias, Cerquiglini (2013), en su artículo Le plurilinguisme à faveur de la science, propone diversos recursos; desde la utilización de herramientas tecnológicas de traducción hasta la oferta de formaciones concentradas en lenguas, pasando por el uso de soportes multilingües en clase y de la intercomprensión como forma de comunicación. Por todo ello, la internacionalización de la educación superior y de la ciencia, pensada a partir de una perspectiva plurilingüe, contribuiría a privilegiar el trabajo en grupos, valorizando la diversidad de culturas científicas y académicas hacia la complementariedad. En este mismo orden de ideas, Berthoud (2013) señala que el plurilingüismo es un antídoto contra el pensamiento único y ayuda a hacer ciencia desde la pluralidad de perspectivas y de saberes. Se abren así caminos por los que avanzar en el espacio iberoamericano, articulando el par lingüístico del español y del portugués, en su enorme diversidad, con las otras lenguas, minoritarias, indígenas y de migración, también presentes. Ampliar así los repertorios lingüísticos en favor de una ciudadanía que reconozca y valorice la pluralidad de conocimientos y saberes. 


\section{Agradecimientos}

Este artículo se inscribe dentro de las acciones de investigación del proyecto Distância e proximidade entre português, francês e outras línguas: potencial da reflexão comparativa coordinado por los profesores Christian Degache (Universidad Federal de Minas Gerais) y Olivier Kraif (Universidad Grenoble-Alpes), aprobado en la convocatoria de cooperación Francia-Brasil CAPES-COFECUB, numéro de proyecto 901/18, para el periodo 2018-2019. Así, esta investigación ha sido financiada por la agencia CAPES (Coordenação de Aperfeiçoamento de Pessoal de Nível Superior) dependiente del Ministerio de Educación de Brasil.

\section{Referencias bibliográficas}

Alas-Martins, S. (2014). A intercompreensão de línguas românicas: proposta propulsora de uma educação plurilíngue. Revista MOARA, 42, 117-126.

Barrios, G. (2006). Minorías lingüísticas y globalización: el caso de la Unión Europea y el Mercosur. Revista Letras, 32, 11-25.

Berthoud, A-C. (2013). Vers une science polyglotte. Cahiers de I'ILSL (Institut de linguistique et des sciences du langage de l'Université de Lausanne), 36, 25-44.

Brasil (s.f.). Escolas de fronteira. Educación integral. Diretoria de Tecnologia da Informação Ministério da Educação (DTI-MEC). Recuperado de https://bit.ly/2ZqLNyY

Brasil (2014). Programa busca a integração de estudantes de áreas de fronteira. Educação Básica, Assessoria de Comunicação Social, Ministério da Educação. Recuperado de https://bit.ly/2ZwkROC

Briceño Ruíz, J. (Ed.). (2011). El Mercosur y las complejidades de la integración regional. Buenos Aires, Argentina: Teseo.

Calvo del OImo, F. J. (2012). La Romania: ébauche d'un carrefour linguistique. Mutatis Mutandis, 5(2), 391-409. Recuperado de https://bit.ly/2zlqYKJ

Calvo del Olmo, F. J. (2018). La experiencia de la UFPR en la inserción de la intercomprensión entre lenguas románicas como herramienta para la formación plurilingüe. Actas del XXVI seminario español en Brasil. Consejería de educación/Embajada de España en Brasil. Madrid: BOE,10-22. Recuperado de https://bit.ly/2ZxpJqm

Candelier, M. et al. (2012, nueva edición). CARAP: un cadre de référence pour les approches plurielles des langues et des cultures. MAREP: Marco de Referencia para los Enfoques Plurales de las Lenguas y de las Culturas. (Centre Européen pour les Langues Vivantes, Ed.). Strasbourg, France: Conseil de I'Europe.

Carullo, A. M. et al. (2007). Intercomprensión en lenguas romances. Propuesta didáctica para el desarrollo de estrategias de lectura plurilingüe. Módulo 2: Hacia el reconocimiento de los esquemas de organización textual. Córdoba: Ediciones del Copista.

Cerquiglini, B. (2013). Le Plurilinguisme en faveur de la Science, Synergies Europe, 8, 11-17. 
Dauzenberg, S. M. (2016). L'intercompréhension au service des stratégies de lecture chez les préadolescents : mise en oeuvre et analyse d'un projet pédagogique. Dissertação de mestrado. Universidade Federal do Paraná, Curitiba. Recuperado de https://bit. ly/2zoVUtn

Degache, C., \& Garbarino, S. (Eds.) (2017). Itinéraires pédagogiques de l'alternance des langues: l'intercompréhension. Grenoble: UGA, Didaskein.

De Sousa, R. (2019). Escola Plurilíngue: Efeitos da Intercompreensão na Aprendizagem de Língua Portuguesa, Curitiba: Appris Editóra.

Erazo-Muñoz, A. (2016). L'intercompréhension dans le contexte plurilingue de l'Université Fédérale de l'intégration Latino-Américaine (UNILA): expériences, contact et interaction plurilingue. Tesis de doctorado, Université Grenoble Alpes, Grenoble, Francia. Recuperado de https://bit.ly/2LdLtOG

Escudé, P., \& Janin, P. (2010). Le point sur l'intercompréhension, clé du plurilinguisme. Paris: Clé international.

Felix, F. M. (2016). Guaranet: Experiências de contato e intercompreensão em guarani, português, espanhol e francês. Dissertação de mestrado. Universidade Federal do Paraná, Curitiba. Recuperado de https://bit.ly/2U7JAXS

Ferreira, L. G. (2018). Política linguística: a historiografia da oferta de línguas estrangeiras no Brasil e a intercompreensão como ferramenta de valorização do plurilinguismo. Dissertação de mestrado, Universidade Federal do Paraná, Curitiba, Brasil. Recuperado de https://bit.ly/2LhOYFY

Gajo, L., \& Fonseca, M. (2014). Didactique du plurilinguisme et intercompréhension intégrée à la lumière d'Euromania. Revista MOARA, 42, 83-98.

García, O. (2011). Bilingual education in the 21st century: a global perspective (2. a ed.). Malden (MA), Estados Unidos de América, Reino Unido e Irlanda del Norte: Wiley-Blackwell.

Hamel, R. E. (2013). El campo de las ciencias y la Educación superior entre el monopolio del inglés y el plurilingüismo: elementos para una política del lenguaje en América Latina. Trabalhos em Lingüística Aplicada, 52(2), 321-384.

Hamel, R. E. (2008). La globalización de las lenguas en el siglo XXI entre la hegemonía del inglés y la diversidad lingüística. En Política Lingüística na América Latina (ALFAL, pp. 45-79). Brasil: Idéia, Editora Universitária.

Kramsch, C. (2014). Teaching Foreign Languages in an era of Globalization. The Modern Language Journal, 98(1), 296-311

Lorenzetti, A., \& Torquato, C. (2016). O Programa Escolas Interculturais de Fronteira (PEIF) como política linguística. Matraga, 23(38), 86-104. Recuperado de https://doi. org/10.12957/matraga

Lüdi, G., \& Py, B. (2003). Etre bilingue. Bern, Allemagne: Peter Lang.

MEC y MECyT (2008). Modelo de ensino comum de zona de fronteira, a partir do desenvolvimento de um Programa para a educação intercultural, com ênfase no ensino do português e do espanhol. Ministério da Educação (MEC) / Ministerio de Educación, Ciencia y Tecnología (MECyT), Brasília / Buenos Aires.

MERCOSUL/MERCOSUR (s.f.). Mercosul Educacional. Recuperado de https://bit.ly/2HsFc05 
MERCOSUL/MERCOSUR (2007). Escuelas de Fronteras. Documento Marco Referencial de Desarrollo Curricular. Programa Escuelas Interculturales Bilingües de Frontera PEBF: Modelo de enseñanza común en escuelas de zona de frontera a partir del desarrollo de un programa para la educación intercultural, con énfasis en la enseñanza de las lenguas predominantes en la región. Recuperado de https://bit.ly/2Ht6ynjl

Moreno Fernández, F. (2007). Qué español enseñar. Madrid: Arco/Libros.

Pérez, A. C., \& Marchiaro, S. (2016). Plurilingüismo en la escuela: aportes para la formación del profesor de lenguas. Revista Digital de Políticas Lingüísticas, 8(8), 141-153. Recuperado de https://bit.ly/2yTpNRO

Pérez, A. C. (2017). Desarrollo de materiales educativos para una educación plurilingüe en escuelas secundarias de la provincia de Córdoba. VII Encontro Internacional de Investigadores de Políticas Linguísticas. Florianópolis, 19 a 21 de setembro de 2017. Universidad Federal de Santa Catarina UFSC. 137-146.

Pinheiro-Mariz, J., \& Lira, M. (2018). A intercompreensão de línguas românicas na formação plurilíngue e intercultural no ensino de línguas estrangeiras para crianças. Letras em revista, 8(01). Recuperado de https://bit. Iy/2Pfu1P6

Sagaz, M. R. (2013). Projeto escolas (interculturais) bilíngues de fronteira: análise de uma ação político linguística. Dissertação de mestrado, Universidade Federal de Santa Catarina, Florianópolis, Brasil. Recuperado de https://bit.ly/2zoENbc

Tancredi, L. (2015). Escolas de fronteira promovem integração cultural e aproximam realidades distantes. Brasil: Ministério da Educação. Recuperado de https://bit.ly/2U5Z2UE

Tassara, G. y Moreno, F. (2007). Manual INTERLAT. Comprensión escrita en portugués, español y francés. Chile: Ediciones Universitarias de Valparaíso. Disponible en https://bit. ly/2zem0iv

UNILA (2013). Plano de Desenvolvimento Institucional (PDI) da Universidade Federal da Integração Latino-Americana (UNILA), relativo ao quinquênio 2013-2017. Ministério da Educação - MEC. Recuperado de https://bit.ly/341/tha 